\title{
Thank you to all our manuscript reviewers in 2014
}

Jan Lubinski ${ }^{1}$, Rodney J Scott ${ }^{2}$, Rolf Sijmons ${ }^{3}$ and Katie Bayliss ${ }^{4^{*}}$

\section{Contributing reviewers}

The editors of Hereditary Cancer in Clinical Practice would like to thank all our reviewers who have contributed to the journal in 2014.

Without the participation of skilful reviewers, no academic journal could succeed, and we are grateful to the committed individuals who have given their time and expertise to the peer review of manuscripts for Hereditary Cancer in Clinical Practice. We look forward to your continued support in 2015.

Annie Anderson

UK

Stefan Aretz

Germany

Patricia Ashton-Prolla
Brazil

Kelly Avery-Kiejda

Australia

Cecelia Bellcross

USA

Lev Berstein

Russia

Morgan Butrick

USA

Daniele Calvano-Mendes

Brazil

Cezary Cybulski

Poland

Lijun Di

China

Katarzyna Durda

Poland

\author{
Maurizio Genuardi \\ Italy
}

Ad Geurts van Kessel

Netherlands

\section{Grey Giddins \\ UK}

Eli Marie Grindedal
Norway

Jacek Gronwald

Poland

Eliete Guerra

Brazil

Parry Guilford

New Zealand

Shirley Hodgson

UK

Veronica Höiom

Sweden

Nicoline Hoogerbrugge

Netherlands

Gill Hubbard

UK
Kay Huebner

USA

Evgeny Imyanitov

Russia

Arvids Irmejs

Latvia

Anna Jakubowska

Poland

Anita Kinney

USA

Marcin Lener

Poland

Noralane Lindor

USA

Jose Lozano

Spain

Henry Lynch

USA

Finlay Macrae

Australia

Diptasri Mandal

USA

\footnotetext{
* Correspondence: katie.bayliss@biomedcentral.com

${ }^{1}$ Pomeranian Medical University, Department of Genetics and

Pathomorphology, Szczecin, Poland

${ }^{2}$ Discipline of Medical Genetics, Faculty of Health, University of Newcastle

and Hunter Medical Research Institute, New South Wales, Australia

${ }^{3}$ Department of Genetics, University Medical Center Groningen,

The Netherlands

${ }^{4}$ BioMed Central, 236 Gray's Inn Road, London WC1X 8HB, United Kingdom
} 
Robert McWilliams

USA

Lawrence Merritt

USA

Steven Narod

Canada

Katherine Nathanson

USA

Maartje Nielsen

Netherlands

Carla Oliveira

Portugal

Domenico Palli

Italy

Edenir Palmero

Brazil
Helle Vendel Petersen

Denmark

\section{Carmen Radecki Breitkopf}

USA

Thangarajan Rajkumar

India

Adelita Ranchor

Australia

Iradj Sobhani

France

Melissa Southey

Australia

Allan Spigelman

Australia

Bente Talseth-Palmer

Australia
Bryony Thompson

Australia

Sergio Toledo

Brazil

Katarzyna Tutlewska

Poland

Alexandre Rezende Vieira USA

Rinse Weersma

Netherlands

Smith Wolf

Pakistan

Michelle Wong-Brown

Australia

Yuan Yuan

China 